\title{
NUCLEAR BINDING ENERGIES AND LIQUID DROP PARAMETERS IN THE EXTENDED THOMAS-FERMI APPROXIMATION *
}

\author{
Y.H. CHU, B.K. JENNINGS ${ }^{1}$ and M. BRACK ${ }^{2}$ \\ Department of Physics, State University of New York, Stony Brook, New York 11794, USA
}

Received 29 March 1977

\begin{abstract}
Using the extended Thomas-Fermi model, we calculate average nuclear hinding energies with Skyrme type effective interactions. The total energy is minimized with respect to variations of the nucleon densities without the use of wave functions or adjustable parameters. We obtain binding energies only $\sim 2-7 \mathrm{MeV}$ higher than selfcunsistenly averaged Hartrec-Fock encrgics. By least-square fits we determine the liquid drop parameters of different effective interactions very accurately. Shell effects are added perturbatively and lead to total energies within $5-10 \mathrm{MeV}$ of the exact Hartree-Fock results.
\end{abstract}

The use of effective nucleon-nucleon interactions as proposed by Skyrme [1] and further based on realistic nucleon forces by Negele and Vautherin [2] permits the total nuclear binding energy in the Hartree-Fock (HF) approximation to be expressed as a functional of the nucleon densities $\rho_{n, p}$, the kinetic energy densities $\tau_{n, p}$ and spin-orbit densities $J_{n, p}[3]$ :

$$
\begin{aligned}
& E_{\mathrm{HF}}=\int \epsilon(r) \mathrm{d}^{3} r \\
& =\int \epsilon\left[\tau_{\mathrm{n}}, \tau_{\mathrm{p}}, J_{\mathrm{n}}, J_{\mathrm{p}}, \rho_{\mathrm{n}}, \rho_{\mathrm{p}}, \nabla \rho_{\mathrm{n}}, \nabla \rho_{\mathrm{p}}, \ldots\right] \mathrm{d}^{3} r, \\
& \rho_{\mathrm{n}, \mathrm{p}}(r)=\sum_{i=1}^{N, Z}\left|\phi_{i}^{\mathrm{n}, \mathrm{p}}(r)\right|^{2}, \tau_{\mathrm{n}, \mathrm{p}}(r)=\sum_{i=1}^{N, Z}\left|\nabla \phi_{i}^{\mathrm{n}, \mathrm{p}}(r)\right|^{2},
\end{aligned}
$$$$
J_{\mathrm{n}, \mathrm{p}}(r)=-\mathrm{i} \sum_{i=1}^{N, Z} \phi_{i}^{* \mathrm{n}, \mathrm{p}}(r)(\nabla \times \nabla) \phi_{i}^{\mathrm{n}, \mathrm{p}}(r) .
$$

Minimizing the energy (1) with respect to variations of the single-particle wavefunctions $\phi_{i}(r)$ lead to the familiar $\mathrm{HF}$ equations [3].

If one expresses $\tau(r)$ and $J(r)^{\ddagger}$ explicitly as functionals of $\rho(r)$, one is led to the so-called energy density formalism [4]. The simplest approximation to

\footnotetext{
^ Work supported in part by U.S.E.R.D.A. contract E(11-1)3001.

1 Supported by the National Research Council of Canada.

2 Present address: Institut Laue-Langevin, Grenoble, France.

₹ The subscripts $n$ and $p$ will be suppressed in the following.
}

$\tau(r)$ is the well-known Thomas-Fermi (TF) approximation which is proportional to $\rho^{5 / 3}(r)$. An extension [5] of the TF model involves gradients of $\rho$ and, when deal. ing with Skyrme interactions, contributions from the nonlocalities of the one body HF Hamiltonian. Writing the latter in the general form

$\hat{H}_{0}=-\frac{\hbar^{2}}{2 m} \nabla \cdot f(r) \nabla+V(r)+i S(r) \cdot(\nabla \times \sigma)$,

where $f(r)$ is the ratio of the free nucleon mass $m$ to the effective mass $m^{*}(r)$ and $\boldsymbol{S}(r)$ the spin-orbit form factor, the explicit extended Thomas-Fermi (ETF) expressions for $\tau$ and $J$ which we presented in ref. [5] are丰:

$$
\begin{aligned}
& \tau_{\text {ETF }}=\left(3 \pi^{2}\right)^{2 / 3} \frac{3}{5} \rho^{5 / 3}+\frac{1}{3} \nabla^{2} \rho+\frac{1}{36} \frac{(\nabla \rho)^{2}}{\rho} \\
& +\frac{1}{6} \frac{\nabla f \cdot \nabla \rho}{f}+\frac{1}{6} \rho \frac{\nabla^{2} f}{f}-\frac{1}{12} \rho \frac{(\nabla f)^{2}}{f^{2}} \\
& +\frac{1}{2}\left(\frac{2 m}{\hbar^{2}}\right)^{2} \rho \frac{(S)^{2}}{f^{2}} \\
& J_{\mathrm{ETF}}=\left(2 m S / \hbar^{2} f\right) \rho .
\end{aligned}
$$

All corrections to the TF term in $\tau_{\mathrm{ETF}}$, as well as $J_{\mathrm{ETF}}$ which has no classical analogon, are linear in $\rho$ and of

\footnotetext{
\# Due to a misprint, a factor of $\rho$ was missing in the sixth term in eq. (7) of ref. [5].
} 
the same order as the Weizsäcker term [6]. Correction terms of the next order are proportional to $\rho^{1 / 3}$; their evaluation is straightforward but very tedious for the general Hamiltonian (2). We have evaluated them [5] for the case $f(r) \equiv 1$ and $S(r)=0$; their contribution to the average kinetic energy in heavy nuclei is $\sim 25-$ $36 \mathrm{MeV}$. In our calculations presented below, they are accounted for by a perturbative correction.

The purpose of our present investigation is to calculate average binding energies of finite nuclei in a variational way without using single-particle wavefunctions. By virtue of the expansions (3), the energy density (1) can be expressed as a functional of the neutron and proton densities only. The average total binding energy of a nucleus may then be calculated by minimizing it with respect to variations of the densities. Our approach differs from ref. [4] mainly in two respects: (1) We use effective interactions which have been previously determined by fitting HF results to experimental data and are not readjusted to make up for deficiencies in the semiclassical expansions. (2) Extensions to the TF approximation are taken into account in a welldefined way which leaves no room for adjustable parameters.

The variation of the densities may be done exactly and the resulting differential equations solved numerically, as done recently by Bohigas et al. [7] leaving out effective masses and spin-orbit potentials. We chose to parametrize $\rho(r)$ in a realistic way and to minimize the energy with respect to the parameters. We used the following function (for each kind of particles);

$\rho(r)=\rho_{0}\left[1+\rho_{1} \exp \left(-r^{2} / \beta^{2}\right)\right] /\{1+\exp [(r-R) / a]\}^{\alpha}$,

restricting ourselves so far to spherical nuclei. The Gaussian term proportional to $\rho_{1}$ allows for a depression of the densities at the center due to Coulomb repulsion. The minimized energy turned out to be insensitive to the parameter $\beta$ and to $\alpha$ in the region $3 \lesssim \alpha$ $\$ 7$. Without introducing an error of more than $0.2 \mathrm{MeV}$ in the total energy, we could take $\beta=0.5 R$ and $\alpha=3.0$ in all nuclei. The variation from $\alpha=1$ to $\alpha=3$, however, decreases the energy in a heavy nucleus by $\sim 8 \mathrm{MeV}$. The parameter $\rho_{0}$ is fixed by the normalization of $\rho(r)$ to the correct particle number $(N$ or $Z$ ). This leaves three parameters per kind of particles or a total of six parameters to be varied. We used a standard computer code for the numerical minimization of the energy with respect to these parameters.

As stated above, the kinetic energy obtained from $\tau_{\text {ETF }}$ in the lead region is about $25 \mathrm{MeV}$ too small; the spin-orbit is overestimated by $J_{\text {ETF }}$ by $\sim 40 \mathrm{MeV}$. Rather than adding further terms to the expansions (3), we account for the lacking convergence by a perturbative correction. To do this we exploit the fact that for a given smooth one body potential $V$, the average single particle energy $\bar{E}_{\mathrm{SP}}=\langle T+V\rangle$ can be approximated to an accuracy of better than $5 \mathrm{MeV}$ by a semiclassical expansion [8] up to second order in $\hbar$. (To get the next order terms in eq. (3) would require the fourth order in the semiclassical expansion.) From our optimized nucleon densities we can easily obtain the corresponding one body potentials $V_{\mathrm{ETF}}$ (including spin-orbit) using the Skyrme formalism [3] and calculate the semiclassical single particle energy $\bar{E}_{\mathrm{SP}}\left[V_{\mathrm{ETF}}\right]$. The expres. sions for $\bar{E}_{\mathrm{SP}}$ including effective mass and spin-orbit are given in ref. [9]; they have been worked out independently by Dworzecka and Moszkowski [10] for the case without spin-orbit. The correction which we include at the end of our variational calculation is then (for each kind of particles)

$$
\begin{aligned}
& \Delta E_{\mathrm{SP}}=\bar{E}_{\mathrm{SP}}\left[V_{\mathrm{ETF}}\right]-\int \mathrm{d}^{3} r\left[\frac{\hbar^{2}}{2 m^{*}(r)} \tau_{\mathrm{ETF}}(r)\right. \\
& \left.+\rho(r) V_{\mathrm{ETF}}(r)\right] .
\end{aligned}
$$

Thus, the error made in the kinetic and spin-orbit energies using eq. (3) is subtracted out perturbatively by adding $\Delta E_{\mathrm{SP}}$ to the total energy; the error in the potential energy is cancelled to lowest order in the error in $\rho$ (see ref. [9] for a more detailed discussion).

In fig. 1 we have plotted the neutron and proton potentials $V_{\mathrm{ETF}}(r)$ for lead (local parts only, including Coulomb), as obtained with the interaction Skyrme III [11]. Shown in fig. 1 are also the exact HF potentials. Inside the nucleus, the semiclassical potentials are seen to fit the HF potentials on the average. Of course they do not exhibit any shell oscillations. In the outer surface region, the fall-off of the (nuclear) potentials $V_{\mathrm{ETF}}$ is somewhat too steep, a deficiency which is typical for the ETF densities and potentials (see also refs. [4,7]).

Our final binding energies, denoted $E_{\mathrm{ETF}}$, are listed in table 1 for four different nuclei including a hypothetical super-heavy nucleus with $A=354(Z=126)$. The interaction Skyrme III [11] was used. The Coulomb 


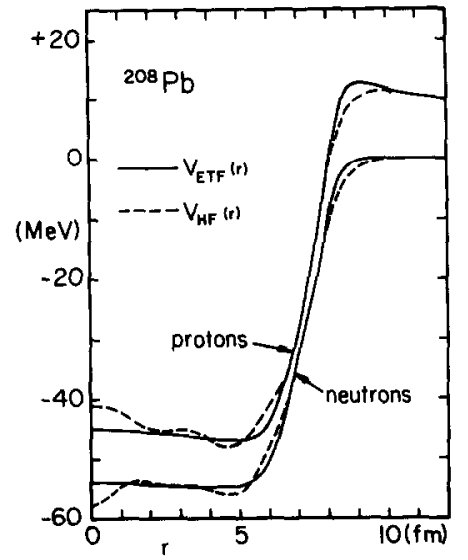

Fig. 1. Local potentials for ${ }^{208} \mathrm{~Pb}$, obtained with the Skyrme III interaction (Coulomb exchange included). Dashed lines: the exact HF potentials. Solid lines: the potentials $V_{\mathrm{ETF}}(r)$ obtained from our optimized densities. The parameters of $\rho$ in eq. (4) are for protons: $\rho_{0}=0.0598 \mathrm{fm}^{-3}, \rho_{1}=-0.119, R=7.58 \mathrm{fm}$, $a=0.46 \mathrm{fm}$; for neutrons: $\rho_{0}=0.0885 \mathrm{fm}^{-3}, \rho_{1}=-0.052$, $R=7.77 \mathrm{fm}, a=0.57 \mathrm{fm}(\alpha=3.0, \beta=0.5 R$ in both cases). The r.m.s. radii are $r_{\mathrm{p}}^{\mathrm{rms}}=5.52 \mathrm{fm}$ and $r_{\mathrm{n}}^{\mathrm{rms}}=5.59 \mathrm{fm}$.

exchange was included in the Slater approximation [2]; no center of mass energy corrections or pairing correlations were included. To check the accuracy of our method, we compare $E_{\text {FTF }}$ to the selfconsistently averaged HF energies $\widetilde{E}_{\mathrm{HF}}$, obtained as in ref. [12] using the Strutinsky method [13]. These energies, given in column 2 of table 1 , are well determined within $\sim 1-2 \mathrm{MeV}$ (except for the case $A=354$, where the limited basis in solving the HF equations leads to an uncertainty of $\sim 2-3 \mathrm{MeV}$ in the Strutinsky-averaged quantities). We see that the energies $E_{\mathrm{ETF}}$ agree within $2-7 \mathrm{MeV}$ with the averaged HF energies. The error, which is positive in all cases, may be due partially to a lack in our parametrization of the densities (4) and partially to the missing convergence of the semiclassical expansions.

It is interesting to see whether we can recover the shell effects in the total energy from our semiclassical results. In ref. [12] it was demonstrated that it is an excellent approximation to add the first-order shellcorrections perturbatively, if the average binding energy and one body potentials have been calculated selfconsistently. We therefore extract the first-order shell-corrections $\delta E_{\mathrm{ETF}}$ from the one body potentials $V_{\text {ETF }}$ in the usual way [13]. The results are shown in
Table 1

Total binding energies and shell-corrections (in MeV) obtained with the Skyrme III force. $E_{\mathrm{ETF}}$ is our present result including the correction $\Delta E_{\text {SP }}$, eq. (5). $\widetilde{E}_{\mathrm{HF}}$ is the selfconsistently averaged and $E_{\mathrm{HF}}$ the exact HF energy. $\langle H\rangle_{\mathrm{ETF}}$ is the expectation value of the Skyrme Hamiltonian, calculated with the s.p. wavefunctions of $V_{\mathrm{ETF}} \delta E_{\mathrm{ETF}}$ and $\delta E_{\mathrm{HF}}$ are the shell-corrections extracted from $V_{E T F}$ and the HF potentials respectively. An asterisk $\left(^{*}\right)$ marks an estimated uncertainty of $2-3 \mathrm{MeV}$ (see text).

\begin{tabular}{lcccccc}
\hline Nucleus & $E_{\mathrm{ETF}}$ & $\tilde{E}_{\mathrm{HF}}$ & $\langle H\rangle_{\mathrm{ETF}}$ & $E_{\mathrm{HF}}$ & $\delta E_{\mathrm{ETF}}$ & $\delta E_{\mathrm{HF}}$ \\
\hline${ }^{40} \mathrm{Ca}$ & -318 & -322 & -320 & -325 & -4 & -3 \\
${ }^{168} \mathrm{Yb}$ & -1332 & -1339 & -1309 & -1317 & +20 & +22 \\
${ }^{208} \mathrm{~Pb}$ & -1593 & -1598 & -1614 & -1619 & -15 & -21 \\
${ }^{354} \mathrm{Xx}$ & -2348 & $-2350^{*}$ & -2347 & -2355 & $-8^{*}$ & $-8^{*}$ \\
\hline
\end{tabular}

table 1 ; we also list there the shell-corrections $\delta E_{\mathrm{HF}}$ obtained from the averaged HF potentials. The agreement between $\delta E_{\mathrm{ETF}}$ and $\delta E_{\mathrm{HF}}$ is reasonable except for lead, where there is a discrepancy of $6 \mathrm{MeV}$.

A different way of including shell effects, used also in ref. [7], which at the same time corrects the deficiency of $V_{\mathrm{ETF}}$ in lowest order, is to calculate the single particle wavefunctions in the potentials $V_{\mathrm{ETF}}$ and to use those to take the expectation value of the two-body (Skyrme) Hamiltonian. This expectation value $\langle H\rangle_{\text {ETF }}$ is also shown in table 1 along with the exact HF energy $E_{\mathrm{HF}}$. The former is seen to be higher than the HF energy by only $5-8 \mathrm{MeV}$ in all cases including lead, where the approximation $E_{\mathrm{ETF}}+\delta E_{\mathrm{ETF}}$ would be $\sim 11 \mathrm{MeV}$ too high. In either way, we can obtain very good approximations to the exact HF energies by solving the Schrödinger equation only once with the potentials $V_{\text {ETF }}$.

The quality of our average binding energies $E_{\mathrm{ETF}}$ allows a very accurate determination of the liquid drop (LD) parameters for a given effective interaction. Since the energy $E_{\mathrm{ETF}}$ by construction is a smooth function of the nucleon numbers $N$ and $Z$, one can fit it with small numerical errors to a LD type mass formula. We chose the following expression:

$$
\begin{aligned}
& E_{\mathrm{ETF}}(N, Z)=a_{\mathrm{v}}\left(1+k_{\mathrm{v}} I^{2}\right) A+a_{\mathrm{s}}\left(1+k_{\mathrm{s}} I^{2}\right) A^{2 / 3} \\
& \quad+a_{\mathrm{c}}\left(1+k_{\mathrm{c}} I^{2}\right) A^{1 / 3}+a_{0}
\end{aligned}
$$

with $A=N+Z$ and $I=(N-Z) / A$. We calculated the 
Table 2

LD parameters (see eq. (6)) for several Skyrme forces and the Moszkowski force (no Coulomb included). $(E / A)_{\text {n.m. is the nuclear }}$ matter binding energy per particle. $a_{\mathrm{S}}^{\prime}$ is the effective surface coefficient for ${ }^{240} \mathrm{Pu}$ defined in the text. Estimated uncertainties are: $0.05 \mathrm{MeV}$ in $a_{\mathrm{V}}, 0.05$ in $k_{\mathrm{V}}, 0.2 \mathrm{MeV}$ in $a_{\mathrm{s}}, 0.2$ in $k_{\mathrm{s}}, 1 \mathrm{MeV}$ in $a_{\mathrm{c}}, \sim 1$ in $k_{\mathrm{c}}$ and $\sim 5 \mathrm{MeV}$ in $a_{0}$. With all the digits given here, the energies $E_{\mathrm{ETF}}$ can be reproduced to within $0.2 \mathrm{MeV}$.

\begin{tabular}{|c|c|c|c|c|c|c|c|c|c|}
\hline & $(E / A)_{\mathrm{n} . \mathrm{m} .}$ & $\begin{array}{l}a_{\mathrm{V}} \\
(\mathrm{MeV})\end{array}$ & $k_{\mathrm{v}}$ & $\begin{array}{l}a_{\mathrm{S}} \\
(\mathrm{MeV})\end{array}$ & $k_{\mathrm{S}}$ & $\begin{array}{l}a_{\mathrm{c}} \\
(\mathrm{MeV})\end{array}$ & $k_{\mathrm{c}}$ & $\begin{array}{l}a_{0} \\
(\mathrm{MeV})\end{array}$ & $\begin{array}{l}a_{\mathrm{S}}^{\prime} \\
(\mathrm{MeV})\end{array}$ \\
\hline SKY II & -16.00 & -15.98 & -2.15 & 19.92 & -4.29 & 11.9 & 6.5 & -12.7 & 15.91 \\
\hline SKY III & -15.87 & -15.83 & -1.77 & 18.64 & -2.54 & 10.6 & 3.3 & -11.7 & 16.42 \\
\hline SKY IV & -15.98 & -15.93 & -1.98 & 19.30 & -5.43 & 14.2 & 8.7 & -15.5 & 14.38 \\
\hline SKY VI & -15.77 & -15.72 & -1.73 & 17.88 & -2.14 & 10.9 & 1.9 & -11.7 & 16.08 \\
\hline MDI & -16.44 & -16.43 & -1.92 & 18.34 & -4.14 & 13.1 & 4.1 & -12.3 & 14.78 \\
\hline
\end{tabular}

energies $E_{\mathrm{ETF}}$ of nuclei in the region $40 \leqslant A \leqslant 400$. Ten nuclei with $N=Z$ and five nuclei with $N \neq Z$ were found sufficient to fit the functional (6) with a very small r.m.s. deviation $\left(\chi_{\mathrm{rms}}<0.01 \mathrm{MeV}\right)$. The constant term $a_{0}$ was found necessary for stabilizing the other coefficients. In those calculations we left out the Coulomb interaction which changes the LD parameters in eq. (6) only little through rearrangement, but makes the numerical fits much more difficult. We want to stress, however, that it is important to include the spinorbit effects which contribute an average of $\sim-2 \mathrm{MeV}$ to the surface energy coefficient $a_{\mathrm{s}}$.

We have investigated the Skyrme interactions SII [3], SIII, IV and VI [14] and Moszkowski's modified $\delta$ interaction (MDI) [14]. In table 2 we present the results of our least-square fits for these interactions. Besides the LD parameters, we also list the nuclear matter binding energies per particle $[11,14]$ which are within $0.05 \mathrm{MeV}$ of our volume energies $a_{\mathrm{v}}$. The volume asymmetry $k_{\mathrm{v}}$ and the surface energy $a_{\mathrm{s}}$ of the force SIII are very close to the Myers-Swiatecki 1966 parameters [15]. The other interactions lead to similar values of $k_{\mathrm{v}}$ and $a_{\mathrm{s}}$. There is a much wider variation in the surface and curvature asymmetry coefficients $k_{\mathrm{s}}$ and $k_{\mathrm{c}}$. The variations in $a_{\mathrm{s}}$ and $k_{\mathrm{s}}$ partially cancel; we list in table 2 the effective surface energies $a_{\mathrm{s}}^{\prime}=a_{\mathrm{s}}\left(1+k_{\mathrm{s}} I^{2}\right)$ for a typical actinide nucleus $\left({ }^{240} \mathrm{Pu}\right)$. For the interactions SII, III and VI the values of $a_{\mathrm{s}}^{\prime}$ are within $\sim 0.5 \mathrm{MeV}$; in the case of SIV which has a rather low effective mass ( $m^{*} \approx 0.5 \mathrm{~m}$ at the origin) and for MDI, $a_{\mathrm{s}}^{\prime}$ is somewhat smaller. The curvature energies $a_{\mathrm{c}}$ are generally larger than the droplet model prediction of Myers and Swiatecki (1969), $a_{\mathrm{c}} \approx 7 \mathrm{MeV}$ [15], by $50-100 \%$; this may be due to the presence of the con- stant term $a_{0}$.

For the interactions SIII and MDI, we did the same kind of fits leaving out the spin-orbit effects in order to compare to the results of Flocard [14] and Dworzecka and Moszkowski [16]. We obtained close agreement to their volume and surface parameters; our curvature terms were again larger for the above reason.

In conclusion, we can state that the ETF model allows one to calculate nuclear binding energies for effective interactions of the Skyrme or Moszkowski type with an accuracy of better than $10 \mathrm{MeV}$, without using wavefunctions or any adjustable parameters. Our method may also be applied to finite range interactions, if the density matrix expansion [2] is used. Shell effects can be added perturbatively by solving once the Schrödinger equation for the variationally obtained one body potentials $V_{\mathrm{ETF}}$; the total energies thereby obtained agree within $\sim 10 \mathrm{MeV}$ with exact HartreeFock energies. Liquid drop parameters for a given interaction can be determined very accurately; a good agreement with earlier determinations is achieved.

\section{References}

[1] T.H.R. Skyrme, Phil. Mag. 1 (1956) 1043.

[2] J.W. Negele and D. Vautherin, Phys. Rev. C5 (1972) 1472.

[3] D. Vautherin and D.M. Brink, Phys. Rev. C5 (1972) 625.

[4] R.J. Lombard, Ann. Phys. (N.Y.) 77 (1973) 380, and references quoted therein.

[5] M. Brack, B.K. Jennings and Y.H. Chu, Phys. Lett. 65B (1976) 1. The missing ref. [13] in that paper is ref. [7] of the present paper.

[6] C.F.v. Weizsäcker, Z. Phys. 96 (1935) 431.

[7] O. Bohigas, X. Campi, H. Krivine and J. Treiner, Phys. Lett. 64B (1976) 381. 
[8] B.K. Jennings, R.K. Bhaduri and M. Brack, Nucl. Phys. A253 (1975) 29; see also B.K. Jennings, Ph.D. thesis, McMaster University, 1976.

[9] Y.H. Chu, Ph.D. thesis, S.U.N.Y. at Stony Brook, 1977.

[10] M. Dworzecka and S.A. Moszkowski, preprint Univ. of Maryland ORO 5126-5, 1976.

[11] H. Flocard, Thèse d'Etat, University of Paris, Orsay, 1975.
[12] M. Brack and P. Quentin, Phys. Lett. 56B (1975) 421.

[13] V.M. Strutinsky, Nucl. Phys. A122 (1968) 1.

[14] D. Ehlers and S.A. Moszkowski, Phys. Rev. C6 (1972) 217.

[15] W.D. Myers and W.J. Swiatecki, Nucl. Phys. 81 (1966) 1; Ann. of Phys. 55 (1969) 395.

[16] M. Dworzecka and S.A. Moszkowski, Phys. Rev. C12 (1975) 619. 